\title{
Developmental Stuttering Screening Instrument: evidence of sensitivity and accuracy measures
}

Mayra Maria Oliveira de Lima ${ }^{1}$ https://orcid.org/0000-0002-3495-7287

Ana Augusta de Andrade Cordeiro' https://orcid.org/0000-0002-8458-3367

Bianca Arruda Manchester de Queiroga ${ }^{1}$ https://orcid.org/0000-0001-5081-924X

Universidade Federal de Pernambuco UFPE, Recife, Pernambuco, Brasil.

Research support source: Fundação de Amparo à Ciência e Tecnologia de Pernambuco - FACEPE.

Conflict of interests: Nonexistent

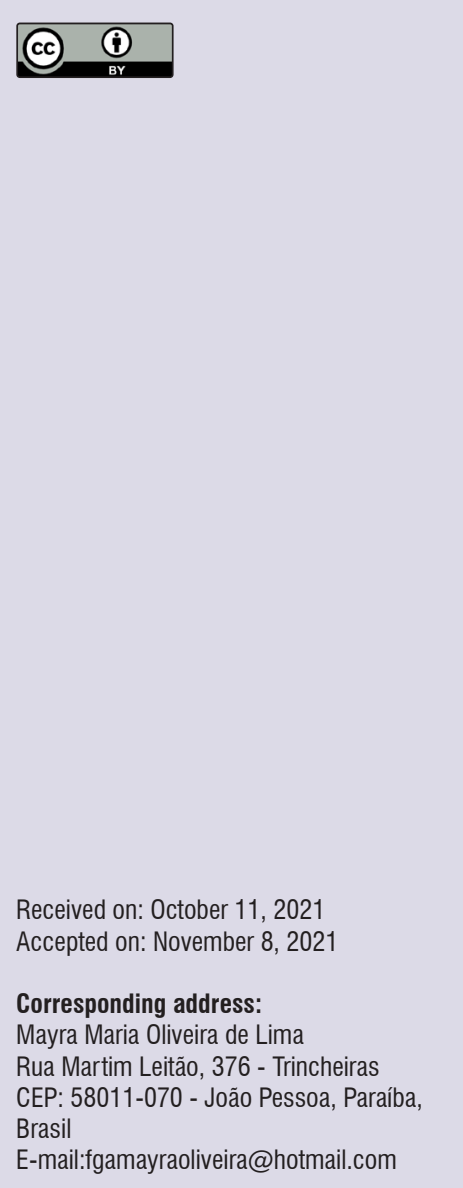

\section{ABSTRACT}

Purpose: to verify the sensitivity and accuracy measures of the Developmental Stuttering Screening Instrument (DSSI).

Methods: the DSSI was administered to 30 parents/guardians of children aged 2 to 5 years and 11 months with and without complaint of stuttering. The instrument administration was timed. The sensitivity analysis used the Weight of Evidence (WoE) binary classification model to verify the strength level of the items. The cutoff scores were established with grouping analysis with the k-means cluster method, based on the minimum and maximum values of each identified group's scores. The data were analyzed with the SPSS statistical software (version 20.0) and were considered significant with $p \leq 0.05$.

Results: the interviews lasted an overall mean of 17 minutes. The WoE model revealed that the items with the greatest predictive strength for risk of stuttering were the social reaction to their speech, the physical concomitants, and the comprehension of the child's speech. The correspondence analysis showed a strong association between "having complaints" and "high total score", as well as between "not having complaints" and "low total score", indicating that the parents' complaints are a factor that leads to high scores in the instrument. "Sex" had little predictive effect for risk. The grouping analysis enabled the stratification of subjects into three risk levels: "not at risk", "under observation", and "at risk".

Conclusion: the instrument presented the first evidence of sensitivity and accuracy measures, thus, making the identification of risk of developmental stuttering in preschoolers, possible.

Keywords: Mass Screening; Stuttering; Protocols; Sensitivity and Specificity; Data Accuracy 


\section{INTRODUCTION}

Stuttering in childhood is one of the most common neurodevelopmental disorders ${ }^{1}$. In approximately $1 \%$ of the world population, atypical speech dysfluency persists throughout life, ever since its onset ${ }^{2}$. Most children are known to overcome their stuttering spontaneously, although $20 \%$ of them remain with it and require follow-up to treat speech fluency ${ }^{3}$.

Aiming to minimize the health damage to child communication and prevent the problem from growing worse with time, preschoolers are often screened to identify, earlier, any speech-language impairments ${ }^{4-6}$. Considering the prevalence and multidimensionality of stuttering, researchers commonly point out the need for practices to screen stuttering in the child population ${ }^{7-9}$. In this sense, the health and education professionals who work with little children are essential to stuttering prevention. With a short and easy-to-use instrument, they can identify the stuttering symptoms in its initial stage and refer them early to specialized speechlanguage clinical assessment ${ }^{10}$. However, no screening instrument has yet been verified in the literature to help these professionals in the early identification of stuttering ${ }^{11}$.

The risk factors for the disorder need to be investigated in children under six years old to know whether they might be acquiring developmental stuttering. The Developmental Stuttering Screening Instrument (DSSI) was developed to further the early identification of preschool children at risk of stuttering ${ }^{12}$. The DSSI was developed to be used with parents and/or guardians by trained health and education professionals who work with children 2 years to 5 years and 11 months old. It is a concise set of 24 questions related to risk factors for stuttering, encompassing the children's overall and communication development and linguistic, motor, and psychosocial aspects related to speech fluency. The instrument has already been analyzed in detail by specialists in the field and gave evidence of content validity and internal consistency ${ }^{12}$.

Continuing the DSSI psychometric evaluations, following its construction and subsequent construct validity, the next stage concerns its capacity to distinguish the target population to whom the instrument was developed. This stage analyzes the sensitivity of the items and the definition of the accuracy measures - i.e., identification of which instrument parameters prove to be most important and which of its cutoff scores make it possible to distinguish the subjects with and without the outcome ${ }^{13}$.

Hence, this study aimed to verify both the sensitivity and the accuracy measures of the DSSI, enabling the instrument scores to be stratified in developmental stuttering risk levels. The hypothesis is that the items that make up the DSSI are sensitive to distinguish preschoolers not at risk of stuttering from those at risk.

\section{METHODS}

The study was approved by the Human Research Ethics Committee of the Federal University of Pernambuco, Brazil, under Certificate of Presentation for Ethical Consideration (CAAE) 00329018.1.0000.5208, with evaluation report no. 3.061.209. Each participant in the study verbally consented to their inclusion after the Informed Consent Form had been read and explained.

\section{Participants}

A total of 30 parents/guardians of children 2 years to 5 years and 11 months old participated in the research, consenting to contribute to it. The research was disseminated on digital media, through which the participants were recruited. It mainly counted with the support from three entities in the field: the Brazilian Institute of Fluency (IBF, in Portuguese), the Brazilian Stuttering Association (Abragagueira), and the Fluency Workshop. A brief sociodemographic questionnaire was used to characterize the sample of respondents and children. This questionnaire gathered information on the subjects' general data, schooling level, occupation, family income, and whether they complained of stuttering. These data are presented in detail in Tables 1 and 2 . 
Table 1. Demographic characteristics of the sample - Parents/Guardians

\begin{tabular}{lcc}
\hline & Group 1 (n=10) & Group 2 (n=20) \\
\hline Sex & - & - \\
Males & $10(100 \%)$ & $20(100 \%)$ \\
Females & & \\
Degree of Kinship & $10(100 \%)$ & $20(100 \%)$ \\
Mother & & \\
Region of the Country & $7(70 \%)$ & $19(95 \%)$ \\
Northeast & $3(30 \%)$ & $1(5 \%)$ \\
Southeast & & \\
Schooling Level & $2(20 \%)$ & $3(15 \%)$ \\
Finished High School & $1(10 \%)$ & $2(10 \%)$ \\
Unfinished Higher education & $7(70 \%)$ & $15(75 \%)$ \\
Finished Higher Education & & \\
Family Income & $1(10 \%)$ & $1(5 \%)$ \\
Up to 1 MW & $4(40 \%)$ & $6(30 \%)$ \\
From 1 to 3 MW & $3(30 \%)$ & $5(25 \%)$ \\
From 3 to 5 MW & $2(20 \%)$ & $3(15 \%)$ \\
From 5 to 8 MW & - & $5(25 \%)$ \\
More than 8 MW & &
\end{tabular}

Caption: $M W=$ minimum wage

Table 2. Demographic characteristics of the sample - Children

\begin{tabular}{lcc}
\hline & Group 1 ( $\mathbf{n = 1 0})$ & Group 2 (n=20) \\
\hline Age & $3: 9(2: 4-5: 6)$ & $3: 5(2: 0-5: 8)$ \\
Sex & & \\
Males & $8(80 \%)$ & $12(60 \%)$ \\
Females & $2(20 \%)$ & $8(40 \%)$ \\
School & & \\
Public & $3(30 \%)$ & $3(15 \%)$ \\
Private & $5(50 \%)$ & $17(85 \%)$ \\
Not yet attending & $2(20 \%)$ & - \\
Schooling Level & & \\
Nursery & $1(10 \%)$ & - \\
Preschool I & $2(20 \%)$ & $8(40 \%)$ \\
Preschool II & $2(20 \%)$ & $6(30 \%)$ \\
Preschool III & $1(10 \%)$ & $3(15 \%)$ \\
Preschool IV & $2(20 \%)$ & $3(15 \%)$ \\
Not yet attending & $2(20 \%)$ & - \\
\hline
\end{tabular}

Based on the participants' complaint or non-complaint of stuttering reported in the interview, they were divided into two groups for data analysis: G1 - group of parents/guardians of the children who complained of stuttering ( $n=10)$; and G2 - group of parents/guardians of the children who did not complain of stuttering $(n=20)$. They were thus divided after collecting enough participants $(n=30)^{14}$ and observing that 10 complained of stuttering, while the others did not. Those included were parents and/or guardians of children aged 2 to 5:11 years with or without complaint of stuttering and without any suspicion or medical diagnosis that might impair the children's overall, language, or speech development. Five participants were excluded from the sample - four of them because on the day of the interview they gave up volunteering, and one because the child had already been submitted to stuttering treatment. 


\section{Materials}

\section{Developmental Stuttering Screening Instrument (DSSI)}

The $\mathrm{DSSI}^{12}$ is a screening instrument aimed at identifying risks of developmental stuttering in preschoolers aged 2 years to 5 years and 11 months, based on the perceptions of the adults responsible for them regarding the child's speech fluency status. It was developed to be used by trained health and education professionals, making possible the early identification of the disorder.

It includes an initial section to record general information on the child, assessor, and respondent. It comprises 24 items presented as questions and distributed into four categories: Category I - Overall and Communication Development (with five items: heredity, peri- and postnatal complications, language development, allergies, and sleep); Category II - Linguistic Aspects (with eight items - forms of stuttering-like dysfluency, non-monosyllabic word repetition, and speech intelligibility); Category III - Motor Aspects of Speech (with five items - incoordination of speech, strained speech, physical concomitants, breathing, and speech speed); and Category IV - Psychosocial Aspects (with six items - the child's response, perception, awareness, and attitude, the social reaction, and communicative pressure).

In Category I, the answer options are "yes", "no", and "I do not know", whose scores are respectively 2 , 0 , and 1 . The other categories assessed the frequency of the risk factors with a 5-point Likert-type scale, in which 1 indicates absence, 5 indicates constant presence, and the other numbers gradually indicate the perception of their frequency (rarely, sometimes, and frequently). The number that best expresses the child's speech fluency at the time is to be marked. The interpretation of the final result is that the higher the total score, the greater the risk of stuttering.

Some room has been provided at the end to write down other qualitative observations related to the parents' complaint but that had not been approached in the instrument and that may help guide future clinical investigations. The instrument also includes a warning that the DSSI does not replace a specialized speechlanguage assessment and that a child whose risk has been identified must be immediately referred to a legally qualified professional for diagnosis and treatment of speech fluency changes problems.

\section{Pamphlet: Fluency Promotion Guidelines}

A digital pamphlet intended for parents and/or guardians provided information on stuttering, especially guidelines to promote fluency and attitudes that help the stuttering child to speak - e.g., respecting the time the child takes to speak, not interrupting them, paying attention to the content of the message, reviewing their lifestyle, avoiding telling them how to speak correctly, speaking more softly and relaxed, and so forth. This material was developed by the Brazilian Institute of Fluency for the 2019 International Stuttering Awareness Day campaign. It is available for free on the institution's website.

\section{Procedures}

The volunteers participated through phone calls. In-person data collection was not possible because it took place in May 2020, amid the worldwide COVID-19 pandemic. Hence, the social isolation measures to prevent the virus spread were respected.

During the phone call, the participants were invited to answer the sociodemographic questionnaire and then the DSSI. The DSSI administration was timed. The data collection procedure was conducted by only one trained speech-language researcher, who also participated in the DSSI construction process.

At the end of the phone call, the participants were given brief qualitative feedback of the risk assessment, as well as guidelines to promote fluency and family attitudes that help the stuttering child. The support material was sent to the parents/guardians via a message application.

\section{Data Analysis}

The data were analyzed with the SPSS statistical software (version 20.0) for Windows. We used descriptive statistics based on measures of central tendency to analyze the length of the instrument administration interviews. The normality distribution of the variables was investigated with the Shapiro-Wilk test.

The DSSI sensitivity was analyzed with the Weight of Evidence $(\mathrm{WoE})^{15}$ binary classification model, considering the 19 items of categories II, III, and IV as independent variables and the complaint as a dependent variable. The first five items were excluded from the discrimination analysis because the type of answer in category $I$ is different from that of the other categories, as the category I variables are categorical 
("yes", "no", or "I do not know"), whereas the other ones are ordinal (from 1 to 5 ).

A two-factor (complaint and sex) analysis of variance (ANOVA), with the total score as a dependent variable, was used to examine the differences between the groups regarding "complaint" and "sex". The category I scores were included in the overall calculation of the instrument in this stage of the analysis. Fisher's Exact test for $2 \times 2$ tables and Cramer's V association were used to detect direct associations between "complaint" and "sex"16. The data were considered significant with $p \leq 0.05$.

To evaluate the association between "complaint" and "total score", the total score was divided into categories: above, below, or equal to the median. Then, a correspondence analysis ${ }^{17}$ was carried out to evaluate how "sex", "complaint", and "total score classification" associated.

Concerning the analysis of accuracy measures, the total score, mean score, and other types of weighted scores were calculated and submitted to a grouping analysis - in which the Euclidean distance, hierarchical clustering, and average linkage method were used ${ }^{18}$. To draw the line separating in groups the possibility of the three stratifications hypothesized in the study - at risk, under observation, and not at risk -, the k-means cluster method ${ }^{17}$ was used, in which the limits were based on the minimum and maximum values obtained by each group in the DSSI. This separation was confirmed afterward with the Tamhane test ${ }^{18}$, in which the dependent variable does not follow normal distribution and heteroscedasticity occurs in the groups' variances. The dendrogram model (tree diagram) was selected, then, to stratify the risk groups based on each child's total DSSI score. The interpretation regarding the risk of stuttering in the dendrogram analysis was that the higher the total score, the greater the likelihood of stuttering. Thus, the subjects could be classified into different groups.

\section{RESULTS}

\section{Instrument administration time}

Overall, the interviews lasted $17 \mathrm{~min}$ and $33 \mathrm{~s}$ on average. As for the instrument administration time per group, the interviews with the parents/guardians without complaints lasted $15 \mathrm{~min}$ and $9 \mathrm{~s}$ on average, while with those who had complaints, it lasted $20 \mathrm{~min}$ and $1 \mathrm{~s}$ on average, as seen in Table 3 . Hence, it took longer to administer the DSSI to the group of parents/ guardians who complained of stuttering (G1) than to the group without complaints (G2).

Table 3. Measures of central tendency of the administration time of the Developmental Stuttering Screening Instrument

\begin{tabular}{cccc}
\hline Measures of Central Tendency & $\begin{array}{c}\text { DSSI administration time in } \\
\text { minutes }\end{array}$ & $\begin{array}{c}\text { DSSI administration time to } \\
\text { G1 in minutes }\end{array}$ & $\begin{array}{c}\text { DSSI administration time to } \\
\text { G2 in minutes }\end{array}$ \\
\hline Mean & 17 min $33 \mathrm{~s}$ & 20 min $01 \mathrm{~s}$ & 15 min $09 \mathrm{~s}$ \\
Minimum & $09 \min 18 \mathrm{~s}$ & 11 min $10 \mathrm{~s}$ & 09 min $18 \mathrm{~s}$ \\
Maximum & $33 \mathrm{~min} 00 \mathrm{~s}$ & $33 \mathrm{~min} 00 \mathrm{~s}$ & $31 \mathrm{~min} 57 \mathrm{~s}$ \\
\hline
\end{tabular}

Captions: DSSI = Developmental Stuttering Screening Instrument; $\min =$ minute; $\mathrm{s}=$ second

\section{Instrument sensitivity}

Concerning instrument sensitivity, Chart 1 shows the predictive power for risk of stuttering of the 19 items of categories II, III, and IV, according to their strength level, from the strongest to the weakest. The items with the greatest predictive effect upon the parents'/guardians' complaints - which therefore were classified as highly contributive to distinguishing the risk of stuttering - were social reaction (C415), physical concomitants (C3|3), and people's comprehension of the children' speech (C2I8). The middle-strength items, with less effect to distinguish the risk, were the respondents' comprehension of the children's speech (in this study, the respondents were exclusively their mothers) (C2I7) and breathing (C3/4). Most of the items (68\%, $n$ $=13$ ) proved to be strong or very strong, that is, with great risk discrimination influence. None of the 19 items was considered weak or very weak. 
Chart 1. List of Developmental Stuttering Screening Instrument items in relation to complaints

\section{Information Value Summary}

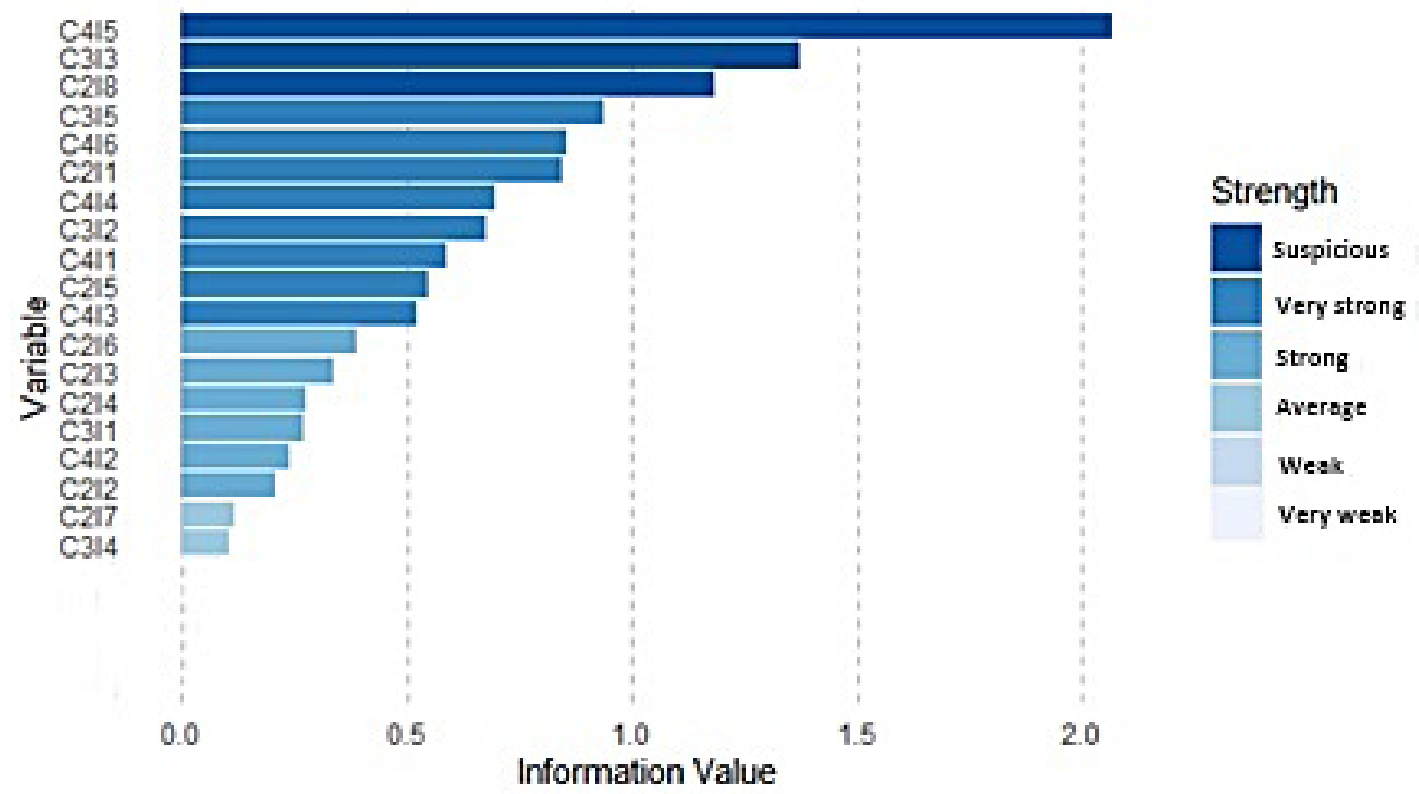

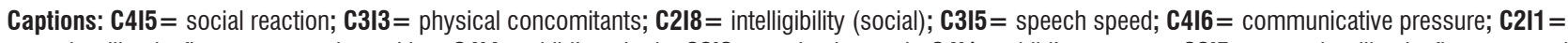
stuttering-like dysfluency - sound repetition; $\mathbf{C} 4 \mathbf{I}$ = child's attitude; $\mathbf{C} 3 \mathbf{I 2}=$ strained speech; $\mathbf{C} 4 \mathbf{I}$ = child's response; C2I5= stuttering-like dysfluency - prolongation; $\mathbf{C 4} \mathbf{3}$ = stuttering awareness; $\mathbf{C 2} \mathbf{6} \mathbf{6}=$ stuttering-like dysfluency - block; $\mathbf{C 2} \mathbf{3} \mathbf{3}=$ stuttering-like dysfluency - monosyllabic word repetition; $\mathbf{C 2} / \mathbf{1 4}=$ other dysfluency - non-monosyllabic word repetition; C3I1 = incoordination of speech; $\mathbf{C} 4 \mathbf{I} \mathbf{2}=$ stuttering perception; $\mathbf{C} \mathbf{2} \mathbf{2}=$ stuttering-like dysfluency - word part repetition; $\mathbf{C} 2 \mathbf{I} \mathbf{7}=$ intelligibility (family environment); C314= breathing.

The analysis of Chart 1 per category reveals that category IV (psychosocial aspects) had the greatest predictive influence for risk in relation to the parents'/ guardians' complaints, as five of its six items were at the "very strong" and "highly suspicious" levels. It is highlighted, though, that all three categories were welldistributed between the strength levels.
The predictive effect of "complaint" on the DSSI total score was significant according to the ANOVA F-test, whereas the influence of "sex" had no significant statistical variation in these scores. Therefore, the presence of complaints is important and must be considered significant to assess the risk of stuttering. "Sex", on the other hand, did not distinguish risk in the group of preschoolers. The results are given in Table 4.

Table 4. Influence of complaint and sex on the instrument's total score

\begin{tabular}{lcccc}
\hline \multirow{2}{*}{ Factors } & Category & \multicolumn{2}{c}{ Total Score } & \multirow{2}{*}{$\begin{array}{c}\text { ANOVA } \\
\text { P-value }\end{array}$} \\
\cline { 3 - 4 } Complaint & Yes & Mean & SD & $<0.001$ \\
\multirow{2}{*}{ Sex } & No* & 37.50 & 5.16 & \\
& Females & 36.80 & 11.41 & 0.618 \\
\hline
\end{tabular}

Captions: $\mathrm{SD}=$ standard deviation; $\mathrm{ANOVA}=$ analysis of variance

Both the Fisher's Exact test and Cramer's V association measure showed that the direct association between "complaint" and "sex" was not significant (the p-value was higher than 0.05 in both). These data reinforce that "sex" had little predictive effect of risk in this sample.

The presence and absence of complaint were assessed alone, associating it to the total score 
- which was divided into categories: above, below, or equal to the median. It is seen in Table 5 that all the children ( $n=10,100 \%$ ) whose mothers complained of stuttering had a high total score (above the total score median), whereas in the group without complaints the predominant result was the opposite: $75 \%(n=15)$ of the children had a total score below the median. The prevalence rate was 3 , which means that high scores are three times more likely to associate with the mother's complaint. In the sample population, this value is probably between 1.47 and 6.14 , with a $95 \%$ confidence. Cramer's V association measure was 0.707 (considered strong), which means that the higher the total score, the more frequent the complaints. In other words, "complaint" and "total score" are positively associated. These results imply that the mother's complaints are a factor that leads to high scores and, therefore, a greater probability of stuttering.

Table 5. Influence of complaint only on the instrument's total score

\begin{tabular}{lccccc}
\hline \multirow{2}{*}{ Dichotomized Total Score } & \multicolumn{2}{c}{ With Complaint } & \multicolumn{2}{c}{ Without Complaint } & Prevalence Rate \\
\cline { 2 - 5 } & $\mathbf{n}$ & $\%$ & $\mathbf{n}$ & $\%$ & and $\mathbf{9 5 \%} \mathbf{~ C l}$ \\
\hline High (> median) & 10 & 100.0 & 5 & 25.0 & 3.00 \\
Low ( $\leq$ median) & 0 & 0.0 & 15 & 75.0 & 1.47 to 6.14 \\
\hline
\end{tabular}

Caption: $\mathrm{Cl}=$ confidence interval.

The correspondence analysis, which evaluated the association of "sex", "complaint", and "total score classification", revealed a strong association between "having complaints" and "high total score", as well as between "not having complaints" and "low total score", as shown in Figure 1. Hence, "complaint" once again points to higher total scores and is, therefore, more associated with stuttering due to the high DSSI scores. "Sex", in its turn, was farther from the other categories, so it can be said that they are little associated with one another. This confirms the low association detected in Fisher's Exact test and Cramer's V measure.

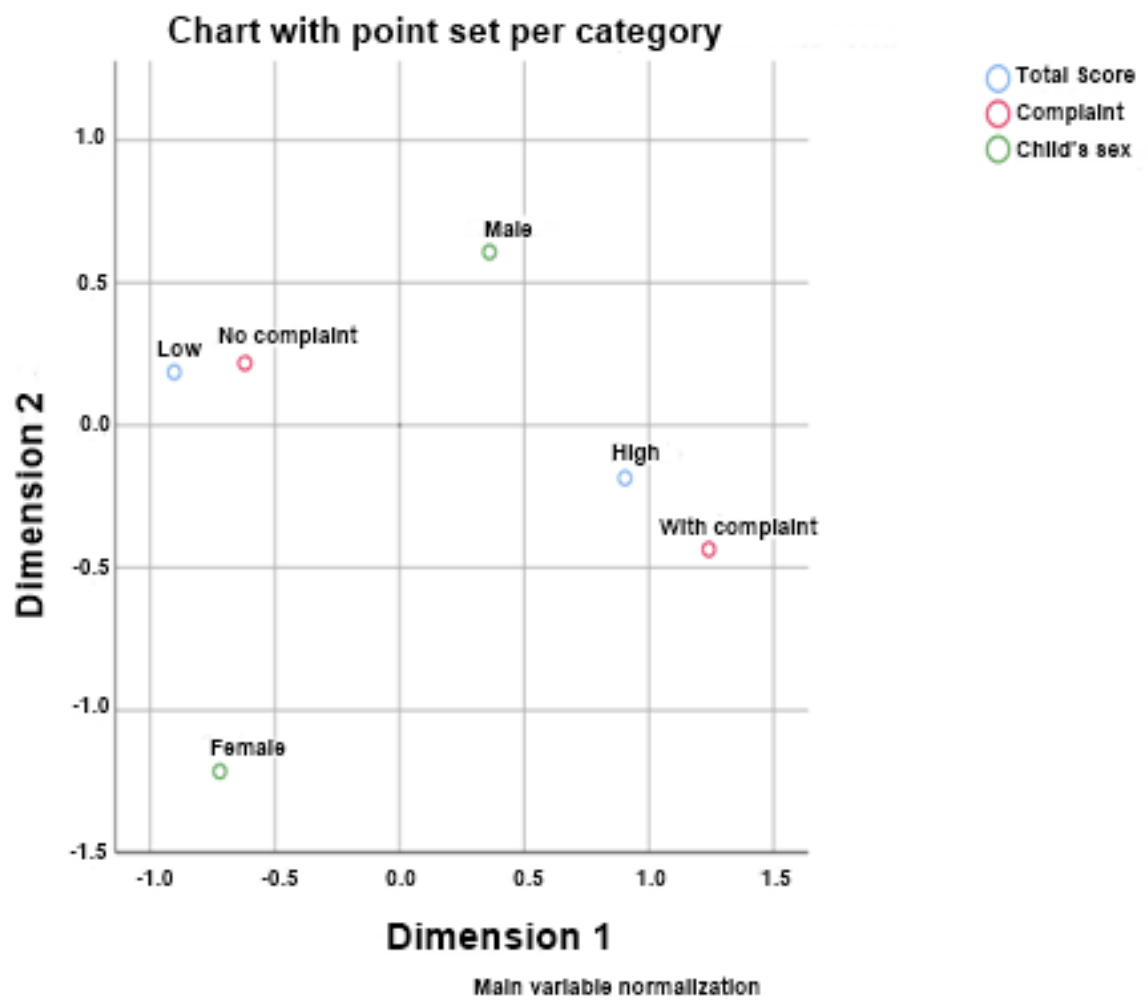

Figure 1. Multiple Correspondence Analysis of Complaint, Child's Sex, and Total Score dichotomized at the median 


\section{Accuracy measures (cutoff scores)}

Many measures were assessed, including each category's weight based on the items' influence detected with the WoE model or with the weight obtained with each category's partial score. Of all these attempts, the dendrogram model (tree diagram) based on the instrument's total score was the one that best stratified the subjects into groups with different risk levels, as shown in Figure 2.

\section{Dendrogram with average linkage (between groups)} Resized distance cluster combination

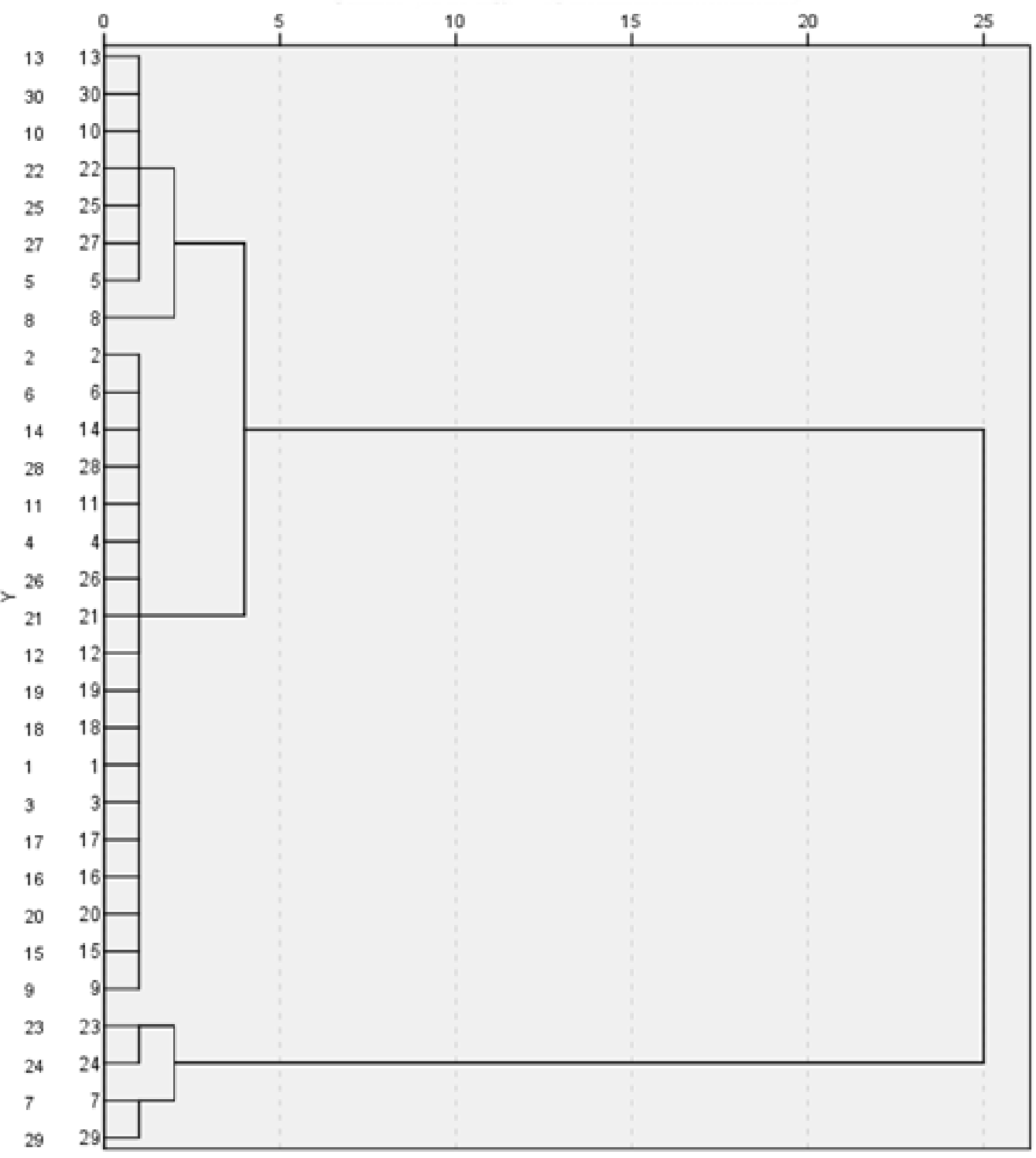

Figure 2. Dendrogram with risk group stratification

The subjects presented in Figure 2 were divided into three groups - G1, G2, and G3 - for a clearer understanding of the data interpretation (Table 6). This division is in itself an indication of the three-level risk stratification of the subjects in the grouping analysis used in the dendrogram. 
Table 6. Classification of the children into groups $\mathrm{G} 1, \mathrm{G} 2$, and $\mathrm{G} 3$

\begin{tabular}{cc}
\hline Groups & Children's number as illustrated in Figure 2, grouped according to the Dendrogram \\
\hline G1 & $1-2-3-4-6-9-11-12-14-15-16-17-18-19-20-21-26-28$ \\
G2 & $5-8-13-10-22-25-27-30$ \\
G3 & $7-23-24-29$ \\
\hline
\end{tabular}

It can be initially seen, in Figure 2, the predominance of two groups, perceived from a perpendicular vertical line at the distance of 5 . As the image narrows to the left, in the column with less than 5 , three horizontal lines indicate the risk stratifications per subject group. Thus, the solution of grouping with the dendrogram lines enabled the subjects to be stratified into the three groups hypothesized in the study - "not at risk", "under observation", and "at risk" -, based on each group's minimum and maximum values (Table 7). Noticeably, the subjects in $\mathrm{G} 3(23,24,7,29)$ are those who had the highest total scores, which potentially characterizes them as in the risk group.

Table 7. Stratification of risk based on the total score

\begin{tabular}{ccccccccccc}
\hline \multirow{2}{*}{ Classification } & \multirow{2}{*}{ N } & \multicolumn{4}{c}{ Total Score } & \multicolumn{3}{c}{ Tamhane Test } & ANOVA \\
\cline { 3 - 8 } & & Min & Max & Mean & SD & Median & $\mathbf{1}$ & $\mathbf{2}$ & $\mathbf{3}$ & Brown-Forsythe \\
\hline Not at risk (G1) & 18 & 30 & 41 & 35.22 & 3.23 & 35.50 & G1 & & & \\
Under observation (G2) & 8 & 45 & 56 & 47.63 & 3.62 & 47.00 & & G2 & & $<0.001$ \\
At risk (G3) & 4 & 70 & 80 & 74.00 & 4.90 & 73.00 & & & G3 & \\
\hline
\end{tabular}

Captions: $N=$ number of subjects; $M i n=$ minimum value; $M a x=$ maximum value; $S D=$ standard deviation; $A N O V A=$ analysis of variance.

Therefore, Table 7 shows that the threshold values - and consequently the cutoffs for each risk level - are as follows: from 24 to 46 points, the subject is classified as "not at risk"; from 47 to 72 points, the subject is classified as "under observation"; and above 73 points, the subject is classified as "at risk" of developmental stuttering.

Considering this study's small sample size for a more precise purpose, it is reasonable to lower to 70 the minimum limit value of the group "at risk". This adjustment brings greater assurance in the detection of risk while other studies on DSSI accuracy measure analysis are not published. Therefore, the primary cutoff score to stratify the three classifications of risk is established as shown in Table 8.

Table 8. Stratification for risk screening with the Developmental Stuttering Screening Instrument

\begin{tabular}{cc}
\hline Classification & Cutoff scores \\
\hline Not at risk & 24 to 46 \\
Under observation & 47 to 69 \\
At risk & 70 to 100 \\
\hline
\end{tabular}

\section{DISCUSSION}

An ideal screening test, different from assessment protocols, must be essentially characterized as easy and quick to use, detecting the greatest number of cases at the lowest possible cost ${ }^{10,19}$. The DSSI took $17 \mathrm{~min}$ and $33 \mathrm{~s}$ on average to be administered, a little more than 2 minutes longer than the maximum time suggested by the Screening Manual of the Brazilian Ministry of Health $^{20}$, which instructs that screening instruments should be administered in up to 15 minutes. A similar time is also mentioned in screenings for developmental delays in children of other countries ${ }^{21}$. However, the time can also be influenced by the complexity of the problem; for instance, when screening patients at risk of dysphagia, the tests take 15 to 20 minutes on average to be administered ${ }^{22}$. Another point to consider is that the research was carried out during the pandemic, in social isolation, requiring the instrument to be administered only via phone calls.

Hence, considering the need for a screening instrument to be quick, the DSSI proved to take a little too long, requiring it to be better calibrated in this aspect. Also, the professionals need to be either trained 
before using it or previously conduct a pilot test to speed its administration. The administration time must be reanalyzed in future research with more assessors involving other trained and calibrated professionals, preferably in person.

On the other hand, having timed the administration showed that the parents/guardians that complained of stuttering took longer to answer the instrument. They usually give more details of aspects of their child's speech, lengthening the interview. This perception may be another risk indicator since the DSSI test tends to be faster in children with no stuttering symptoms.

This research verified that the DSSI has a rather low administration cost, as it can be conducted even via phone call, not requiring printed material. In in-person screening procedures, the assessor needs only a pen and a copy of the instrument, which is only three sheets long. As for easiness, the instrument was administered by only one professional, who had already been trained as she also helped construct it. It is suggested that a screening instrument cost as little as possible, be financially cheap, and be quick and easy to use - which are characteristics that make it different from the other assessment and diagnostic clinical tests ${ }^{19}$. In this regard, the DSSI is an economically accessible screening instrument, while the easiness criterion needs to be better verified in future administration analyses to compare the assessors' perception.

Concerning the instrument sensitivity, three items were identified with the highest level of prediction strength for stuttering in the perception of parents/ guardians - namely: social reaction to the child's speech (psychosocial aspect), physical concomitants (motor aspect of speech), and people's comprehension of the child's speech (linguistic aspect). These items refer to the effects of stuttering on the child, though not necessarily to the cause of the disorder. The striking presence of stuttering dysfluency can lead to an atypical effort to speak to the point of the child having to combine it with facial or body movements, such as blinking and moving the head and arms. This worries the interlocutor, who in turn may pay more attention to the manner how the child is speaking than the content of the message ${ }^{23}$. These difficulties in the communicative interaction with stuttering children are related to the three most predictive items found in the study, which also reinforces the concern of the parents'/ guardians' complaints.

The most alarming factors for the parents/guardians in the DSSI interview were the functioning of speech fluency and the psychosocial impact on the communication of those who stutter, regarding both themselves and others around them. For them, the consequences of a speech that is not much functional nor easy to understand, expressed in a quite distractive manner, are more significant than the disorder itself. These findings are similar to those of studies that investigate the parents' reactions to their children's stuttering, which are mostly cognitive, emotional, and behavioral of which, the emotional aspect has the greatest impact on the mothers, who comprised this study's sample group ${ }^{24}$.

Based on the parents' reports, the preschoolers' speech intelligibility was a strong risk factor, which may be influenced by the fact that, in this phase, the child is still in the process of developing the language and acquiring the phonetic-phonological system ${ }^{25}$. Thus, not only is the child still maturing their expressive and receptive vocabulary, the phoneme articulation motor processing, and the control of speech sounds, but also they present stuttering-like dysfluency. This way, natural development processes mingle with atypical speech events, interfering with its continuity, making it more difficult and less clear when they express themselves - that is, with an unintelligible character and functional impairment.

The psychosocial aspect was the most frequent category in the parents' perception of their children's speech fluency. This category is directly related to the quality of life of the person who stutters. The concern with a child's stuttered speech is related to how they cope, are coping, or will cope with their speech difficulty and how their social environment reacts or will react to the disorder. In other words, the possible negative psychosocial impact due to changes in their children's speech fluency is what reinforces the parents' complaints - which is also one of the main reasons that encourage them to seek clinical attention. It is not only because of the disorder itself, but also its possible consequences. It is not without reason that they feel this way, as developmental stuttering may be associated with a decrease in psychosocial (behavioral, emotional, and social) well-being beginning at 3 years old $^{26}$. Hence, reacting and being reacted upon with negative feelings (such as sadness, frustration, shame, and anxiety) and behaviors (such as little eye contact, interrupted speech, and inadequate attention to the spoken message) may become part of the everyday life of stuttering children ${ }^{24}$. 
All the three DSSI categories were well-distributed between the strength levels. None of the items in the linguistic, speech motor, and psychosocial aspects were considered weak or very weak in the item sensitivity analysis. This result may be explained with the multifactorial dynamic pathways theory of the development of stuttering ${ }^{3}$. The brain of a stuttering child since a very young age presents different neuroanatomical physiology from that of fluent children ${ }^{27}$. It develops with complex, nonlinear interactions with unstable control, impairing the speech sensory-motor processes - which, with time, is strongly conditioned by linguistic and emotional factors ${ }^{3}$. Hence, the factors that make up the DSSI categories II, III, and IV adequately fulfill the role of listing and identifying the risks of stuttering with onset in childhood, helping to early detect the disorder.

In addition to demonstrating the items that better distinguish the risk of stuttering, another equally relevant result of the study was the parents' complaints. The mothers' complaints were a factor that increased the scores and, as a result, tripled the likelihood of risk of stuttering. The parents of stuttering children can distinguish a speech with stuttering-like dysfluency from one with common dysfluency ${ }^{3}$. Therefore, their concern regarding the child's speech must be taken seriously in any assessment. In the group of eight children who were classified as "under observation", the parents had expressed their complaint of either stuttering or other language disorders. This shows that the DSSI may be sensitive to detect other communication difficulties as well. The DSSI assessor to whom an affirmative complaint of stuttering is presented or who notices that the total score has increased to the "under observation" level - for the instrument points to increased risk as the value goes up - must take professional action. It is recommended that even if the final value does not classify the child as "at risk", the assessor provide fluency promotion instructions and refer them to an in-depth assessment. Thus, they avoid any negligence in child health care.

The child's sex had no statistical significance in any of the analyses of the study. The sex did not distinguish risk in the sample group possibly because the epidemiologic proportion of stuttering onset among preschoolers is approximately 1.5:1 between boys and girls, respectively ${ }^{3}$. It is a phase in which stuttering has not yet become chronic; it can either persist or be overcome. This disorder's male/female prevalence relationship only changes more evidently in school-age children when the stuttering becomes more chronic unless it is treated ${ }^{3}$.

The study has some limitations that must be solved in future research. The small sample size due to the short data collection time and the global health crisis and the lack of a gold standard assessment in the field to diagnose children of the risk group were the main gaps that withheld greater credibility from the results. It is also inferred that the DSSI administration time can be optimized. Moreover, the instrument can be adapted to a digital version, possibly making its administration and interpretation even faster, besides reducing its cost. The DSSI must be further improved, continuing the psychometric validation processes, and furnishing other analyses and perceptions from different assessors.

Thus, the DSSI can be a prophylactic resource in stuttering. Once administered to each child, it helps in early identification when the risk is detected. Not only that, but it can also be a preventive tool against the disorder, as it is directly related to the need for fluency promotion guidelines at the end of the instrument.

\section{CONCLUSION}

This study presented the first evidence of DSSI sensitivity and accuracy measures in the identification of the risk of developmental stuttering. The DSSI proved to be sensitive, registering a strong predictive value in most of its items. The presence of parents'/ guardians' complaints can triple the likelihood of risk and must, therefore, be considered in the final conduct. Hence, the DSSI can be used by health and education professionals as a stuttering screening tool, making the early identification of the disorder, possible. For further research, it is suggested that (1) the sample size be larger; (2) the children at risk be compared with a clinical diagnosis; (3) the instrument be administered by more professionals to obtain other psychometric validation analyses; (4) the instrument be administered in a digital format for comparison.

\section{ACKNOWLEDGMENT}

Gratitude is extended to the Pernambuco Science and Technology Support Foundation (FACEPE).

\section{REFERENCES}

1. Onslow M, O'Brian S. Management of childhood stuttering. J Paediatr Child Health. 2013;49(2):E112-5. 
2. Yairi E. Future research and clinical directions in stuttering: global considerations. 2016. Available at: https://www.stutteringhelp.org/future-research.

3. Smith A, Weber C. How stuttering develops: the multifactorial dynamic pathways theory. J Speech, Lang Hear Res. 2017;60(9):2483-505.

4. Mohamadi O, Rahimi-Madiseh $M$, Sedehi $M$. The prevalence of stuttering, voice disorders, and speech sound disorders in preeschoolers in Shahrekord, Iran. Int J Child Youth Family Stud. 2016;7(3/4):456-71.

5. Thapa KB, Okalidou A, Anastasiadou S. Teachers' screening estimations of speechlanguage impairments in primary school children in Nepal. Int J Lang Commun Disord. 2016;51(3):310-27.

6. Shimada M, Toyomura A, Fuji T, Minami T. Children who stutter at 3 years of age: a community-based study. J Fluency Disord. 2018;56:45-54.

7. Ward D. Risk factors and stuttering: evaluating the evidence for clinicians. J Fluency Disord. 2013;38(2):134-40.

8. Oliveira CMC, Souza HA, Santos AC, Cunha $D$, Giacheti CM. Fatores de risco na gagueira desenvolvimental familial e isolada. Rev. CEFAC. 2011;13(2):205-213.

9. Howell P. Screening school-aged children for risk of stuttering. J Fluency Disord. 2013;38(2):102-23.

10. Mirawdeli $A$, Howell $P$. Is it necessary to assess fluent symptoms, duration of dysfluent events, and physical concomitants when identifying children who have speech difficulties? Clin Linguist Phon. 2016;30(9):696-719.

11. Lima MMO. Identificação de risco para a gagueira do desenvolvimento: elaboração e validação de um instrumento de rastreio [thesis]. Recife (PE): Universidade Federal de Pernambuco; 2020.

12. Lima MMO, Cordeiro AAA, Queiroga BAM. Developmental Stuttering Screening Instrument: development and content validation. Rev. CEFAC. 2021;23(1):1-19.

13. Pernambuco L, Espelt $A$, Magalhães Junior HV, Lima KC. Recommendations for elaboration, transcultural adaptation and validation process of tests in Speech, Hearing and Language Pathology. CoDAS. 2017;29(3):e20160217.

14. Siddiqi N. Credit risk scorecards: developing and implementing intelligent credit scoring. New Jersey: John Wiley \& Sons, 2006.
15. Siegel S, Castellan Jr NJ. Estatística não-paramétrica para ciências do comportamento. São Paulo: Bookman (Artmed), 2006.

16. Greenacre M. Correspondence analysis in practice. New York: Chapman \& Hall/CRC, 2007.

17. Johnson RA, Wichern DW. Applied multivariate statistical analysis. New Jersey: Englewood Cliffs, 2007.

18. Goulart BNG, Chiari BM. Testes de rastreamento $x$ testes de diagnóstico: atualidades no contexto da atuação fonoaudiológica. Pró-Fono R. Atual. Cient. 2007;19(2):223-32.

19. Brasil, Ministério da Saúde. Secretaria de Atenção à Saúde. Departamento de Atenção Básica. Rastreamento. Série A. Normas e Manuais Técnicos Cadernos de Atenção Primária. 2010;(29):1-95.

20. Best Practice Advocacy Centre New Zealand. The use of Screening Tests. 2010, p.2-7. Available at: https://bpac.org.nz/BT/2010/July/docs/best_test_ jul2010_screening_pages2-7.pdf.

21. Etges CL, Scheeren B, Gomes E, Barbosa LR. Screening tools for dysphagia: a systematic review. CoDAS. 2014;26(5):343-9.

22. Eichorn N, Fabus R. Assessment of stuttering disorders in children and adults chapter. In: Cyndi S-R, Renee F, editors. A Guide to clinical assessment and professional report writing in speech-language pathology. New York: Cengage Learning, 2012. p.347-98.

23. Salehpoor A, Latifi Z, Tohidast SA. Evaluating parents' reactions to children's stuttering using a Persian version of Reaction to Speech Disfluency Scale. Int J Pediatr Otorhinolaryngol. 2020;134:110076.

24. Ceron MI, Gubiani MB, Oliveira CR. Keske-Soares M. Normative features of phoneme acquisition in Brazilian portuguese. Folia Phoniatr Logop. 2020;72(3):228-41.

25. McAllister J. Behavioural, emotional and social development of children who stutter. J Fluency Disord. 2016;50:23-32.

26. Chang S-E, Angstadt M, Chow HM, Etchell AC, Garnett EO, Choo AL et al. Anomalous network architecture of the resting brain in children who stutter. J Fluency Disord. 2018;55:46-67.

27. Weber-Fox C, Wray AH, Arnold H. Early childhood stuttering and electrophysiological indices of language processing. J Fluency Disord. 2013;38(2):206-21. 